\title{
Bildung für nachhaltige Entwicklung inklusiv mit dem „Whole School Approach" verankern - Beispiele aus der Grundschulpraxis
}

Mit diesem Projekt wurde in Kooperation zwischen Behinderung und Entwicklungszusammenarbeit e.V. (bezev) und zwei Grundschulen der Frage nachgegangen, wie Inklusives Lernen und Bildung für nachhaltige Entwicklung (BNE) mit dem Whole School Approach (WSA) im Lernort Schule verankert werden kann. Kann BNE inklusiv als Motor für Schulentwicklung dienen? Wie kann Unterricht entlang einer BNE inklusiv gestaltet werden?

Die aus dem Projekt hervorgegangene Handreichung „BNE inklusiv als Aufgabe der ganzen Schule“ (Behinderung und Entwicklungszusammenarbeit e.V., 2019) ist das Ergebnis eines dreijährigen Prozesses und verbindet wissenschaftliche Ansätze einer BNE und inklusiven Lernens mit konkreten Anregungen aus dem Schulalltag. Ziel dieser Handreichung ist es, Lehrkräfte und Schulleitungen bei ihren individuellen Schulentwicklungsprozessen sowie bei der Entwicklung von Unterrichts- und Lernangeboten so zu unterstützen, dass nachhaltige Entwicklung und Inklusion konsequent berücksichtigt werden.

Im partizipativen Prozess mit den beiden Grundschulen hat bezev zu verschiedenen Handlungsfeldern von Schule Orientierungsfragen entwickelt. Als Grundlagen dienten hier der Index für Inklusion (Boban \& Hinz, 2003), der Orientierungsrahmen für den Lernbereich Globale Entwicklung (KMK \& BMZ, 2016) sowie das Drei-Wege-Modell (Rolff, 2012, S. 14ff.). Die Orientierungsfragen mündeten in ein Analyse- raster (Abb. 1), welches das Kollegium dabei unterstützen kann, BNE inklusiv und systematisch an der eigenen Schule zu verankern.

Dieses Analyseraster diente den beiden Grundschulen für eine Bestandsanalyse (Wo stehen wir? Was machen wir schon, was läuft gut und wo besteht Handlungsbedarf?). Mit dieser Herangehensweise wurde zum einen die Selbstreflexion der Schulen unterstützt, zum anderen konnten so relevante Handlungsbereiche im Lernort Schule identifiziert werden.

So konnten beide Schulen eine individuell angepasste Mindmap (Abb. 2) erstellen und Differenzierungen der eigenen Handlungsebenen einer BNE und Inklusion herausarbeiten. In enger Anlehnung an den Index für Inklusion wurden so gezielte Fragen entlang einer BNE entwickelt, die Schulen sowohl als Anregung zur Umsetzung als auch zur Weiterentwicklung für eigene, schulspezifische Fragen für den Schulentwicklungsprozess dienen können. Mit diesem Projekt wurde erstmalig eine Lanze gebrochen, BNE und Inklusion in Kooperation zwischen Schule und außerschulischen Partnern aus dem Feld BNE mit dem WSA an Grundschulen systematisch anzugehen. Inklusion sowie Schulentwicklung sind stets als Prozess zu verstehen. Das vorgestellte Projekt und die Handreichung „BNE inklusiv als Aufgabe der ganzen Schule“ sollen dazu anregen, in diesen Prozess einzusteigen und so auch zur inklusiven Umsetzung des Nationalen Aktionsplans BNE beizutragen.

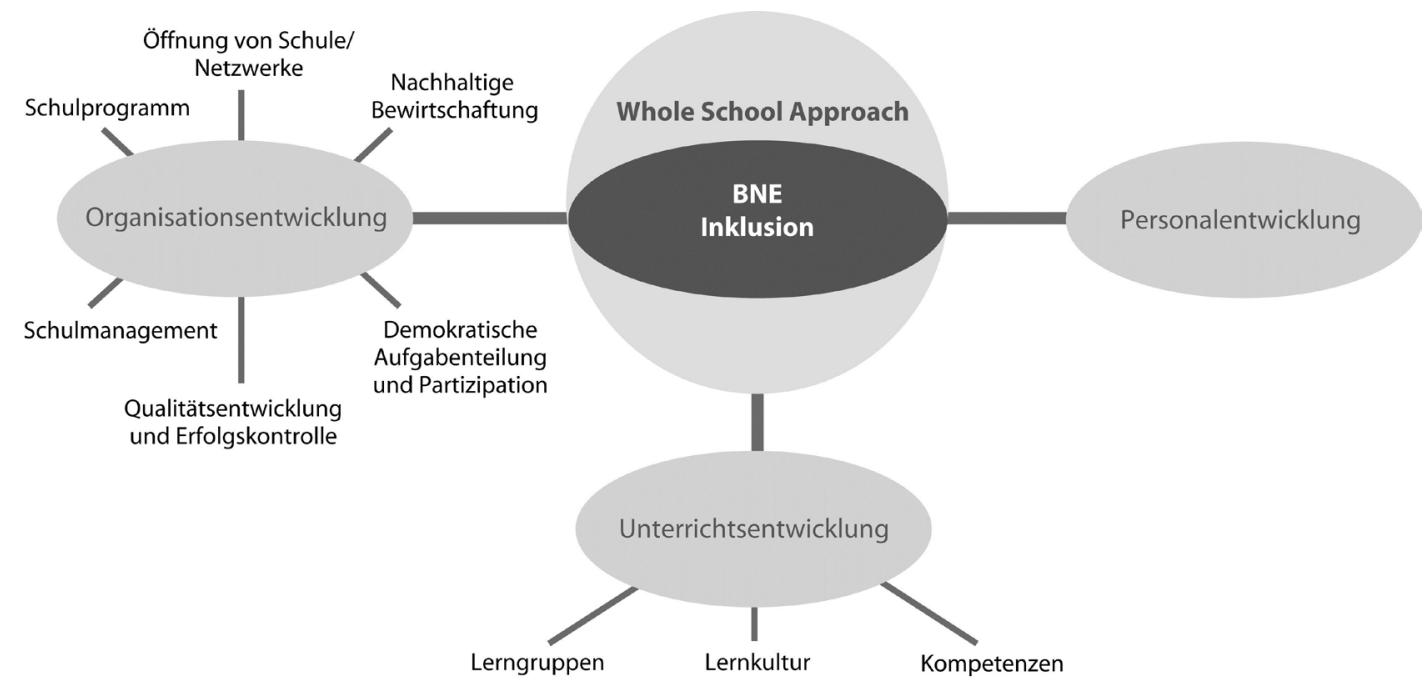

Abb. 1: Analyseraster, Quelle: Behinderung und Entwicklungszusammenarbeit e. V., 2019, S. 16 


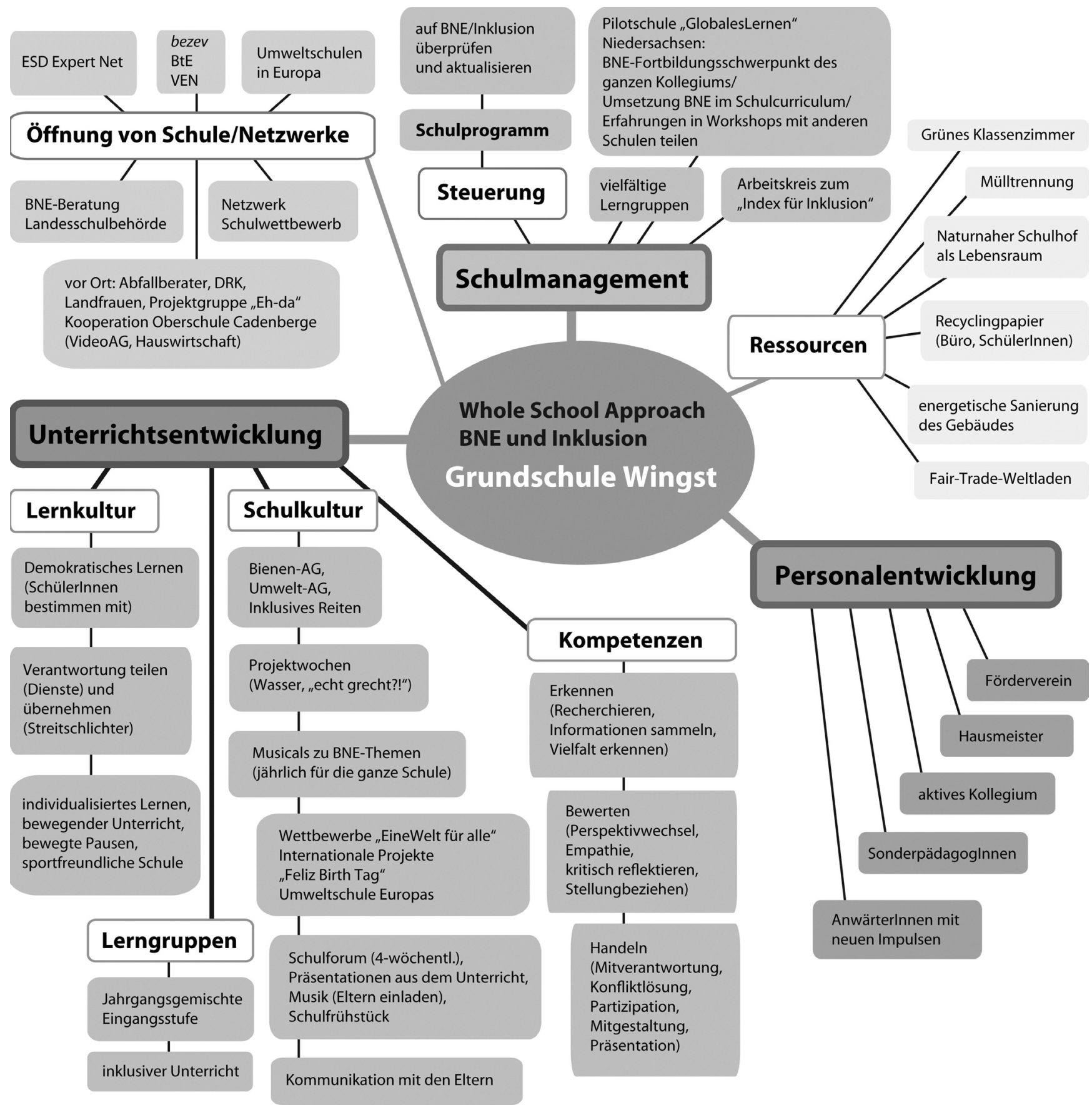

Abb. 2: Mindmap der Schule am Wingster Wald, Quelle: Behinderung und Entwicklungszusammenarbeit e. V., 2019, S. 18

\section{Literatur}

Behinderung und Entwicklungszusammenarbeit e. V. (2019). Bildung für nachhaltige Entwicklung inklusiv als Aufgabe der ganzen Schule. Essen. Zugriff am 18.12.2019 https://www.bezev.de/de/home/service-und-bestellungen/

Boban, I. \& Hinz, A. (2003). Index für Inklusion. Lernen und Teilhabe in der Schule der Vielfalt entwickeln. Zugriff am 06.12.2019 https://www.eenet.org.uk/resources/ docs/Index\%20German.pdf

KMK - Kultusministerkonferenz \& BMZ - Bundesministerium für wirtschaftliche Zusammenarbeit und Entwicklung (2016). Orientierungsrahmen für den Lernbereich Globale Entwicklung (2. aktualisierte und erweiterte Auflage). Bonn: Cornelsen.

Rolff, H.-G. (2012). Schulentwicklung kompakt. Modelle, Instrumente, Perspektiven. Weinheim \& Basel: Beltz.

Judith Altenbockum, Behinderung und Entwicklungszusammenarbeit e. V. (bezev) \& Katarina Roncevic, Greenpeace e. V. doi.org/10.31244/zep.2020.01.07 